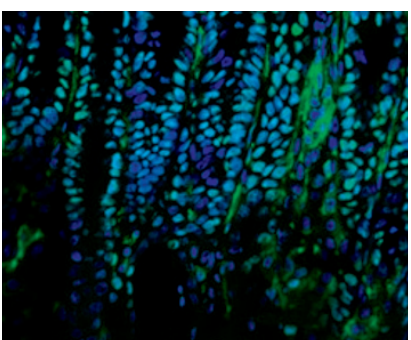

p 129

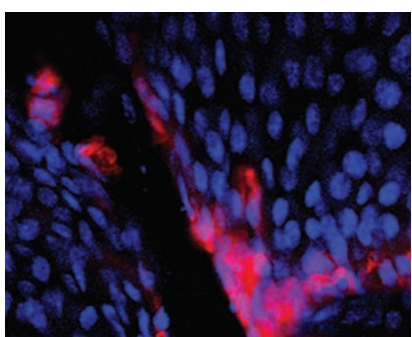

p 173

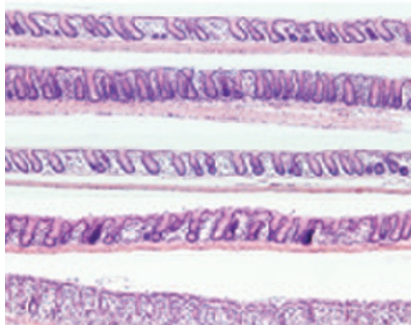

p 194

\section{Periodontal disease and rheumatoid arthritis}

Dermot Farquharson and colleagues provide an intriguing summary of studies addressing potential mechanisms by which periodontal disease may contribute to the initiation and progression of rheumatoid arthritis. See page 112

\section{Foreskin T-cell subsets}

Jessica Prodger and co-workers demonstrate that the foreskin - the primary site of HIV acquisition in uncircumcised heterosexual men-represents a proinflammatory milieu that is enriched for HIVsusceptible T-cell subsets. See page 121

\section{Targeting TPL-2 kinase in colitis}

Maria Lawrenz and colleagues demonstrate a positive role for the tumor-progression locus-2/extracellular signal-related kinase pathway independent of nuclear factor-кB activation in dextran sulfate sodium-induced colitis, using both knockout mice and pharmacological inhibitors. See page 129

\section{Cytokines affect fibrocyte function}

Alberto Bellini and colleagues found that fibrocytes from asthma patients that were stimulated with interleukin (IL)-4 and IL-13 induced the production of extracellular matrix components whereas fibrocytes stimulated with IL-17A proliferated and released proinflammatory factors. See page 140

\section{Targeting of antigens to CD103}

Using a mouse model of asthma, Monika Semmrich and co-workers observed that in vivo administration of ovalbumin-coupled anti-CD103 antibodies results in immunological tolerance or immunity, depending on the absence or presence of an adjuvant, respectively, and that tracheal application prevented airway inflammation. See page 150

\section{Granzyme-producing Tregs in RSV}

Jens Loebbermann and colleagues present data supporting a critical role for granzyme B expressed by regulatory $T$ cells in controlling lung inflammation during experimental respiratory syncytial virus infection in mice. See page 161

\section{Novel sensing of HSV-1 by IFI-16}

Christopher Conrady and colleagues demonstrate that IFI-16/p204, a DNA sensor that induces interferon-regulatory factor-3 signaling, is important for innate immunity to herpes simplex virus- 1 and viral containment in both the corneal and vaginal epithelium. See page 173

\section{CD103+ DCs make TSLP}

Ilaria Spadoni and coauthors show that thymic stromal lymphopoietin is produced by $\mathrm{CD}_{103}{ }^{+}$dendritic cells in the steady state and acts on T cells to reduce their capacity to produce interleukin-17 and enhance the development of Foxp3 ${ }^{+}$T cells. See page 184

\section{Myeloid cell Myd88 in epithelial cell injury}

Using cell-specific deletion of Myd88, Nicole Malvin and colleagues found that the epithelial cell response to injury following dextran sulfate sodium-induced colitis is dependent on Myd88 signaling in myeloid cells. See page 194

\section{Negative regulation of mucus production}

Kyoung Seob Song and co-workers report that the transcript factor c-Ets1 suppressed interleukin-1 $\beta$ induced MUC5AC gene expression by disrupting the interaction of nuclear factor- $\mathrm{KB}$ with $\mathrm{CREB}$ on the MUC5AC promoter, thus identifying a novel negative regulatory pathway for mucus production relevant to airway inflammation. See page 207

\section{M cells for prion entry}

Using a mouse model, David Donaldson and colleagues found that $\mathrm{M}$-cell depletion prevents early prion accumulation on follicular dendritic cells in Peyer's patches, as well as neuroinvasion and disease development. See page 216 